\title{
Imaging evaluation for the diagnosis and management of complications of gender-affirming surgeries
}

\author{
Justin T. Stowell ${ }^{1}$, Allie M. Metcalfe ${ }^{1}$, Priyanka Jha ${ }^{2}$ \\ ${ }^{1}$ Department of Radiology, Mayo Clinic in Florida, Jacksonville, FL, USA; ${ }^{2}$ Department of Radiology, University of California San Francisco, San \\ Francisco, CA, USA \\ Contributions: (I) Conception and design: JT Stowell, P Jha; (II) Administrative support: All authors; (III) Provision of study materials or patients: \\ JT Stowell, P Jha; (IV) Collection and assembly of data: JT Stowell; (V) Data analysis and interpretation: All authors; (VI) Manuscript writing: All \\ authors; (VII) Final approval of manuscript: All authors. \\ Correspondence to: Justin T. Stowell, MD. 4500 San Pablo Rd., Jacksonville, FL 32224, USA. Email: stowell.justin@mayo.edu.
}

\begin{abstract}
Gender-affirming surgeries (GS) allow transgender individuals to align their physical sexual characteristics with their gender identity, which can result in profound changes to native anatomy. Medical imaging is a useful tool for evaluation of patients who have undergone or plan to pursue GS. Given the complex nature of some GS, complications may arise. The choice of imaging modality can be guided by the clinically suspected complications. For example, urethral complications of phalloplasty are best evaluated with fluoroscopic urethrography. Pelvic magnetic resonance imaging provides detailed depiction of pelvic neo-anatomy after vaginoplasty. Many GS involve the creation of vascular pedicles for tissue grafts, which are at risk of thrombosis and graft ischemia. Doppler ultrasound and computed tomography (CT) angiography are important for diagnosis of these dreaded complications. Moreover, interventional radiologists may participate in endovascular treatments for such complications. Various imaging modalities may assist the surgeon in the postoperative evaluation of patients with suspected complications after GS, and imaging protocol modifications may be required to improve diagnostic accuracy. For example, rectal or neovaginal contrast material may be necessary to ensure accurate imaging evaluation, such as delineation of fistulas. Working together, surgeons and radiologists can ensure accurate imaging assessment while accommodating for patient comfort.
\end{abstract}

Keywords: Transgender; gender-affirming surgery; phalloplasty; vaginoplasty; mammography; computed tomography (CT); magnetic resonance imaging (MRI); urethrography

Submitted Sep 15, 2020. Accepted for publication Jan 29, 2021.

doi: 10.21037/atm-20-6429

View this article at: http://dx.doi.org/10.21037/atm-20-6429

\section{Introduction}

Gender-affirming surgeries (GS) allow transgender individuals to align their physical sexual characteristics with their gender identity, which can result in profound changes to native anatomy. Broadly, these surgeries can be classified as masculinizing or feminizing surgeries. Given the complex nature of some GS, complications may arise. Various imaging modalities may assist the surgeon in the postoperative evaluation of patients with suspected complications after GS. For example, urethral complications of phalloplasty are best evaluated with fluoroscopic urethrography. Surgeons should work in concert with radiologists to ensure the correct imaging studies are performed so as to maximize diagnostic accuracy all the while maintaining patient comfort. In this review, case-based clinical scenarios have been used to illustrate the most appropriate image work-up of suspected complications of GS.

\section{Masculinizing surgeries}

GS for transmasculine persons include chest masculinization, 
hysterectomy with or without oophorectomy, vaginectomy and neophallus construction via phalloplasty or metoidioplasty (1). These procedures may be further augmented by testicular implants and inflatable penile prostheses. Patients may choose to pursue components of these surgeries and not undergo all of the above surgeries. For example, a patient may desire phalloplasty without pursuing hysterectomy and vaginectomy. The choice of imaging modality can be guided by the clinically suspected complications. Creation of a long neo-urethra is a critical step procedure during phalloplasty and urethral elongation, which can be complicated by urethrocutaneous fistula, anastomotic leak, and urethral strictures or stenosis. Urethral complications are best evaluated with fluoroscopic urethrography. Retrograde urethrography (RUG) allows for neourethral evaluation where contrast is instilled through the neourethral meatus to opacify the urethra. RUG can be accompanied by voiding cystourethrography (VCUG), which can be performed by retrograde filling of the bladder from the neourethra. Rarely, the bladder can be distended in an antegrade fashion in patients who have indwelling suprapubic catheters. Contrast-enhanced computed tomography (CT) or magnetic resonance (MR) are helpful in evaluation and comprehensive characterization of many complications. Noncontrast technique can be useful for localizing hematomas, though contrast is necessary if an active site of bleeding needs to be identified. Ultrasound (US) evaluation with Doppler allows for the evaluation of vessel integrity in the immediate postoperative state or the diagnosis and potential drainage of localized abscess or hematoma.

\section{Hysterectomy/oophorectomy}

Masculinizing genital surgeries are usually performed in a staged fashion, with hysterectomy performed several months prior to phallic construction (2). Laparoscopic or robot-assisted hysterectomy are now favored given lower rates of perioperative complications and challenges that may be faced in this population with a vaginal hematoma, abscess, vaginal cuff dehiscence, and rarely bladder perforation, which are best assessed with contrast-enhanced CT or MR. Targeted US can be utilized to localize postsurgical wound infections and fluid collections.

\section{Phalloplasty}

Following hysterectomy, creation of a neophallus may be elected by the transmasculine patient. Two main techniques for creation of a neophallus are phalloplasty and metoidioplasty. Metoidioplasty is the simpler of the two approaches and has a lower complication rate. It involves hormone-induced clitoral hypertrophy and urethral lengthening to form a neophallus and is often elected by patients who wish to avoid the morbidity of phalloplasty (3). Phalloplasty involves the creation of a neophallus using grafts and is pursued by patients who desire standing micturition and penetrative intercourse. These can be categorized broadly as free flap and pedicled flap techniques, with radial artery free forearm flap (RFFF) being the most commonly utilized technique. Pedicled anterolateral thigh flap (ALT) and other flap techniques have largely fallen out of favor given higher rates of complications, although the ALT flap technique allows for more bulk compared to RFFF technique (4). If pedicled flaps are utilized, preoperative imaging with CT or MR angiography (CTA, MRA) for vascular mapping can be employed (5). Given the complexity of phalloplasty operations, complications are quite common and may occur in the immediate postoperative period to several years following surgery. CT is the most appropriate imaging modality in the immediate post-operative period, with additional role for magnetic resonance imaging (MRI) for delayed-onset and long-term complications.

\section{Phalloplasty complications \\ Vascular compromise, ischemia, and graft failure}

\section{(I) Case example}

A 34-year-old transmasculine patient with RFFF phalloplasty 10 years prior presented for emergent care with complaint of hematuria and no pulsation in his neophallus. The patient had been traveling by car for 3 days when his symptoms began. On physical exam, the neophallus was pale and cool to the touch. What imaging tools may be helpful for vascular assessment?

\section{(II) Discussion}

Doppler US is the most appropriate technique to evaluate for vascularity and perfusion of the neophallus. In the immediate postoperative period, patients are monitored closely with Doppler US or commercially available tissue oxygenation meters as there is increased risk for thrombosis at vascular anastomoses during this period (6). Thrombosis is evidenced as lack of vascular flow and tissue vascularity on color Doppler and spectral Doppler interrogation (Figure 1). CTA can demonstrate arterial occlusion as nonenhancement of the vascular stalk to the neophallus. The neophallus may show reduced enhancement due to lack 

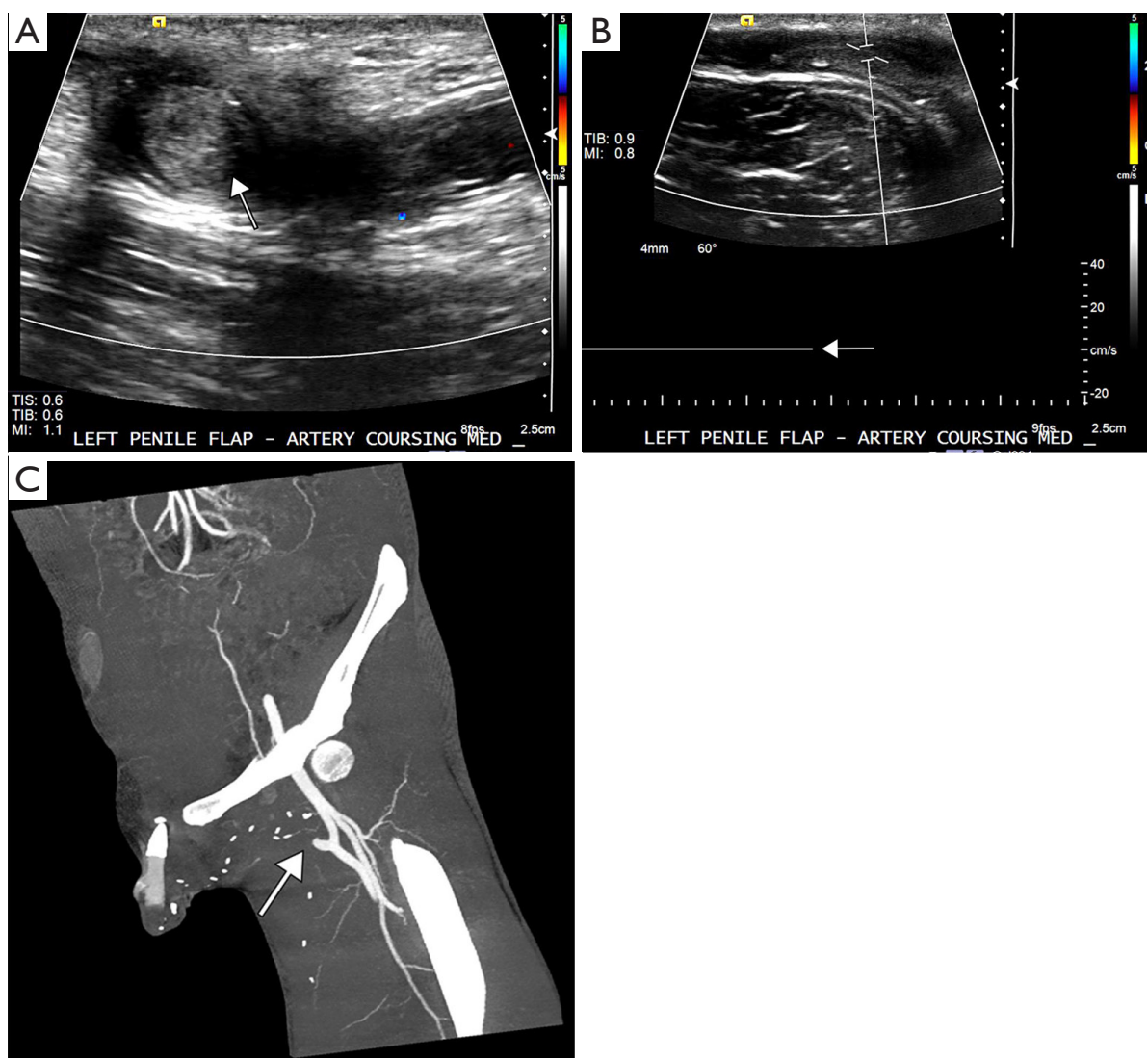

Figure 1 Vascular compromise, acute arterial thrombosis and graft ischemia. A 34-year-old transmasculine person presented with pale and pulseless phallus, after a prolonged car journey, suggestive of phalloplasty ischemia. (A) Doppler ultrasound showed lack of color flow in the phallus and no flow in its arterial supply, indicating thrombosis (arrow) of the left inferior epigastric artery pedicle just distal to the origin. (B) Spectral Doppler confirmed lack of arterial waveforms in the pedicle (arrow). (C) CT angiogram (coronal oblique, maximum intensity projection reformat) demonstrates abrupt cut off at the expected location of the inferior epigastric artery at the pedicle, confirming arterial thrombosis (arrow) and lack of arterial perfusion in the phalloplasty. Post-surgical changes with multiple radiopaque clips are seen in the left groin at the site where the phalloplasty flap was mobilized.

of perfusion, as well. Ischemic graft compromise may be remote to surgery as in this example. Overall, US and CTA are useful imaging tools for diagnosing suspected vascular compromise including thrombosis, pseudoaneurysm, and arteriovenous fistula. Active hemorrhage and hematoma can be seen in the immediate post-operative period, as well. In some cases, invasive catheter angiography may be required for revascularization including catheter-directed thrombolysis or stent placement if required.

\section{Hematoma, seroma, and abscess}

\section{(I) Case example}

A 73-year-old transmasculine patient developed progressive groin swelling and pain 5 days after ALT flap phalloplasty. The patient was afebrile. Physical exam revealed soft tissue swelling in the right inguinal region and at the base of the phalloplasty graft. There was no redness, fluctuance, or crepitus. What is the next best step for evaluation?

\section{(II) Discussion}

CT with contrast is the most appropriate imaging modality for immediate post-operative complications. Complications in the first days to weeks following surgery are usually related to hematoma, seroma, or infection and are best evaluated with contrast-enhanced CT or with MR if there are contraindications to contrast-enhanced CT (Figure 2). A suspected superficial infection may be evaluated with targeted US or CT to look for drainable fluid collections which can be treated with image-guided aspiration or drain placement. While non-contrast CT is sufficient for 

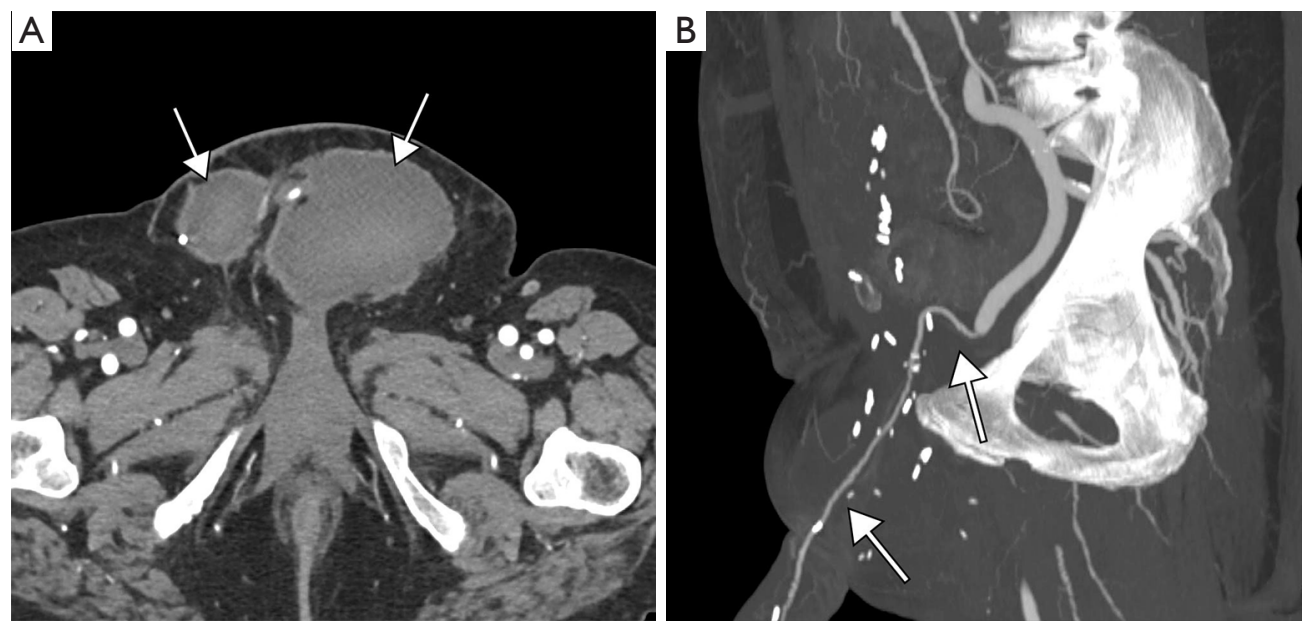

Figure 2 Postoperative hematoma after anterior lateral thigh (ALT) flap phalloplasty. A 74-year-old transmasculine patient with groin swelling and pain 5 days after ALT flap phalloplasty. Computed tomographic angiography (CTA) was performed to assess graft vessel patency and for evidence of active hemorrhage. (A) Axial CTA shows two large hematomas (arrows) along the vascular pedicle. No active extravasation was seen. (B). Coronal maximum intensity images from the CTA demonstrate patent graft artery (arrows).

detecting and localizing hematomas, contrast is necessary for evaluating active bleeding, which is identified as sites of active extravasation of intravenously administered contrast material on arterial phase imaging, followed by "blooming" on venous and delayed phases. Performance of arterial and delayed phase imaging also allows for comprehensive assessment of arterial and venous patency.

Neouretbral complications (stricture, leak, or fistula)

(I) Case example

A 20-year-old transmasculine patient recently underwent RFFF phalloplasty. His post-operative course was uncomplicated and the indwelling suprapubic catheter is ready to be removed. What is the most appropriate imaging modality for evaluation of neourethral integrity prior to removal of the catheter?

\section{(II) Discussion}

Neourethral complications are best evaluated by RUG or VCUG. Phalloplasty complications are most often related to the neourethra. Most complications occur at the anastomotic sites between the native and midportion urethra and midportion and phalloplasty urethra due to tenuous blood supply to these sites. In the immediate postoperative period, anastomotic leaks and iatrogenic fistulous connections are prevalent. In the subacute to remote period, strictures, contained leaks, sinus tracts, and fistula formation are encountered (7). In patients who do not receive vaginectomy, fistulous communication between the neourethra and native vagina can occur. This complication has been described in nearly half of patients who present for treatment of neourethral strictures and can lead to long term complications such as pelvic pain, post void dribbling and chronic urinary tract infections (8). Post vaginectomy patients can develop urethro-cutaneous and urethro-rectal fistulae.

The gold standard for neourethral evaluation is fluoroscopic urethrography (VCUG and RUG) (1). VCUG is generally the test of choice for immediate post-operative evaluation as suprapubic catheters are generally placed at the time of surgery allowing for access to the urinary bladder with minimal manipulation of the neourethra (Figure 3). Patients who no longer have suprapubic catheters in place undergo RUG (9). Urethrography readily identifies most of the complications, of which urethrocutaneous fistulas occur with the highest frequency and an incidence ranging from $15 \%$ to $70 \%$ (10). Strictures and leaks can be readily identified on RUG. Information from VCUG further augments the information from RUG by providing functional information about the areas of narrowing. When strictures are seen on RUG, VCUG can provide valuable information, whether these areas of narrowing are causing upstream obstruction and impeding the urinary stream. When performed concomitantly, RUG is performed first and then the bladder is distended followed by the VCUG portion of the exam.

\section{Erectile devices and testicular implants}

(I) Case example

A 44-year-old transmasculine patient who had undergone 


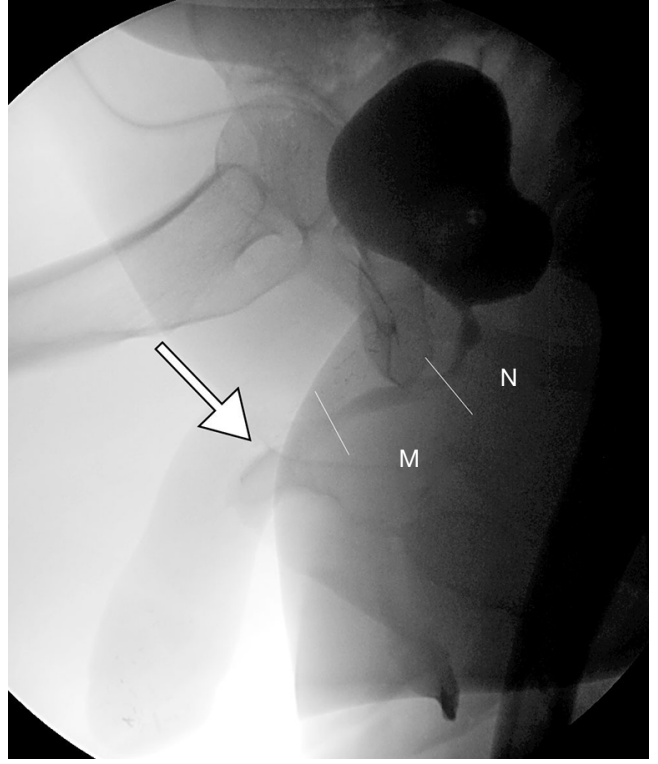

Figure 3 Neourethral leak. A 20-year-old transmasculine patient underwent RFFF phalloplasty. Voiding cystourethrogram was performed after bladder contrast administration via a suprapubic catheter to assess neourethral integrity. (A) VCUG shows extravasation of contrast (arrow) at the anastomosis between the urethral segment in the phalloplasty and midportion of the urethra (M). $\mathrm{N}$ = native urethra. The distal phalloplasty urethra does not opacify, consistent with a high-grade stricture. RFFF, radial artery free forearm flap; VCUG, voiding cystourethrography.

RFFF phalloplasty and subsequent erectile device and testicular implant placement complained of pain in the neophallus. He reported that the device still functioned appropriately. Physical exam showed a normal appearing neophallus with functioning hydraulic erectile device. There was no external evidence of infection or hematoma. What imaging modality can be used for further evaluation of the patient's pain?

\section{(II) Discussion}

CT followed by MRI are most commonly employed for evaluation of erectile device and testicular prosthesis related complications (Figure 4). Protocol modifications in patients who have malfunctioning hydraulic erectile devices may include both inflated and deflated views. For transmasculine patients who intend to engage in penetrative intercourse, semi-rigid or inflatable/hydraulic erectile devices are implanted. This is often the final step in construction of the neophallus with complication rates ranging from $23 \%$ to $70 \%(11,12)$. Implant complications
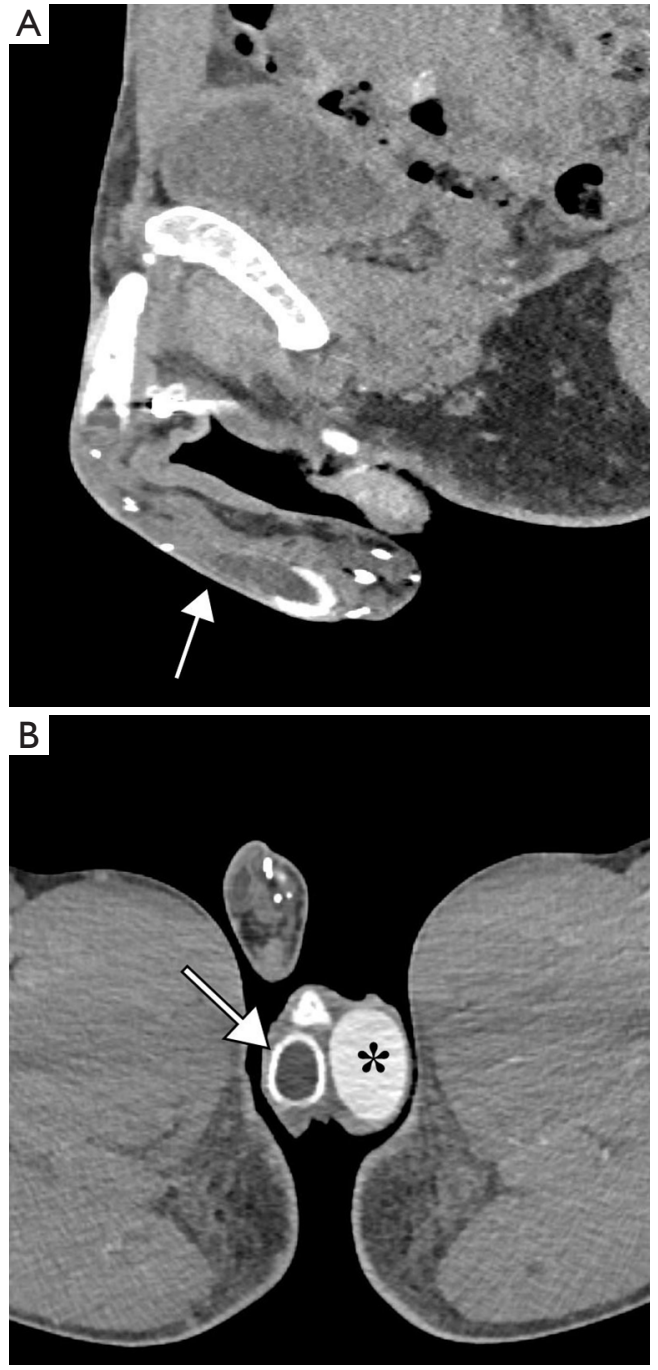

Figure 4 Normal hydraulic erectile device and testicular prostheses. A 44-year-old transmasculine patient complained of neophallus pain after placement of a hydraulic erectile device and testicular prostheses. (A) Computed tomography (CT) showed intact prosthesis elements including the hydraulic cylinders (arrow) that extend along the phalloplasty shaft. (B) The hydraulic pump (arrow) component of the device may be placed in the neoscrotum to simulate a testis. Note the adjacent high-attenuation silicone implant $\left(^{*}\right)$.

(malposition, protrusion, infection) may occur at higher rates among transmasculine patients than for cisgender males. This is due to lack of corpora cavernosa for device insertion/support and lack of supportive fascial layers in the neophallus. On imaging, the implant components can be seen eroding through the skin and fluid collections can be 


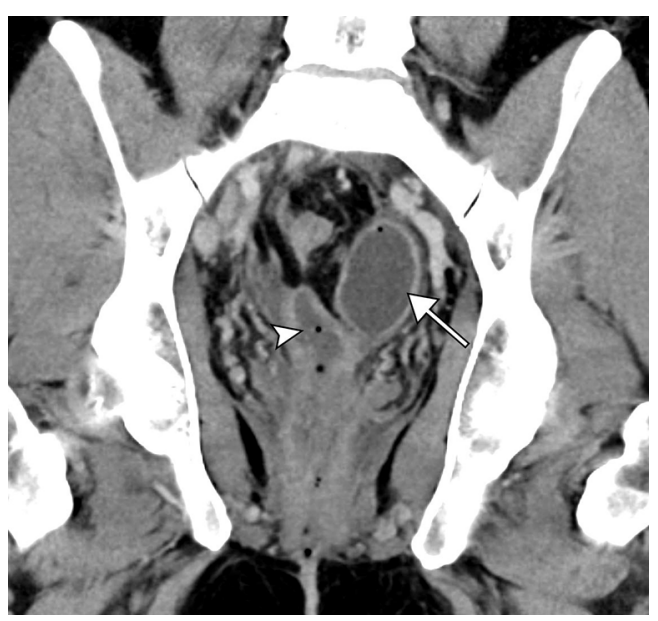

Figure 5 Abscess after vaginoplasty. A 33-year-old transfeminine patient complained of pelvic pain and fever 1 week after undergoing penoscrotal inversion vaginoplasty. Coronal contrastenhanced computed tomography (CT) shows a rim-enhancing fluid collection (arrow) containing small bubbles of gas. The collection is located deep in the pelvis, just superior and to the left of the distal portion of the neovagina (arrowhead).

present adjacent to them due to superinfection and abscess formation. Extensive scar tissue in the neophallus may also predispose the transmasculine patient to infection $(11,12)$. Testicular prostheses utilized for creation of aesthetic genitalia after scrotoplasty may contain a silicone or saline filler (2). The silicone prosthesis appear hyperattenuating on CT and saline infused reservoirs are of fluid attenuation. On T2-weighted fluid-sensitive images, both silicone and saline appear hyperintense. Although usually not necessary for testicular prosthesis, silicone-specific MRI sequences are available to look for free silicone, if needed. The hydraulic pump for erectile devices may be substituted to simulate a testis. Complications associated with testicular prostheses are uncommon but include pain, hematoma, infection, or implant dislocation and extrusion. Occasionally, testicular implants can also obstruct urinary flow by external pressure on the neourethra $(11,13)$.

\section{Feminizing surgeries}

Feminizing surgeries for transfeminine persons may involve breast augmentation or genital reconstruction with penectomy and vaginoplasty. Penoscrotal inversion vaginoplasty (PIV) is the most common surgical approach utilized these days, with enteric vaginoplasty reserved for salvage options for candidates with failed PIV and those with inadequate penoscrotal skin available for vaginoplasty (as can happen with patients who received pre-pubertal hormone blockers). Complications of vaginoplasty vary by temporal proximity to surgery, with woundrelated complications (hematoma, bleeding, abscess, flap dehiscence) occurring in the first days to weeks after surgery, while neovaginal stenosis, fistulas and prolapse tend to occur months to years after surgery (2). Erectile tissue (corpora spongiosum and cavernosum) is typically removed at surgery, and residual erectile tissue may become a source of bleeding or cause urethral stenosis. The engorged tissue may cause distress to some patients during sexual arousal. Cross-sectional imaging with CT and MR is preferred for evaluation of these conditions. Imaging protocols may be modified to include a neovaginal dilator instrument which would allow for adequate tissue distension, especially when evaluating for neovaginal stenosis. Instillation of neovaginal or rectal contrast material allows for better detection and characterization of fistulas. Dynamic pelvic MR may be helpful to show prolapse. Although urethral complications are less commonly encountered with vaginoplasty, when suspected, fluoroscopy could be considered.

\section{Vaginoplasty complications}

Early post-operative complications (hematoma, abscess, bladder injury)

\section{(I) Case example}

A 33-year-old transfeminine patient presented with pelvic pain, fevers, and night sweats one week after her penoscrotal vaginoplasty procedure. She denied neovaginal bleeding, discharge, and foul odors. Bladder and bowel movements were normal. Laboratory evaluation was positive for leukocytosis. Physical exam showed normal early postoperative changes in the perineum. There was no evidence of soft tissue abscess. What is the next most appropriate step to determine the presence of a deep pelvic abscess?

\section{(II) Discussion}

Contrast-enhanced CT is most suitable for imaging of immediate post-operative complications. Wound-related complications are most frequent in the early post-operative period following vaginoplasty, with wound infection and abscess occurring in up to $17.0 \%$ of cases (14). Patients may present with pain and constitutional symptoms suggestive of infection. The differential diagnosis in this setting includes flap necrosis, abscess, and potentially fistula. 


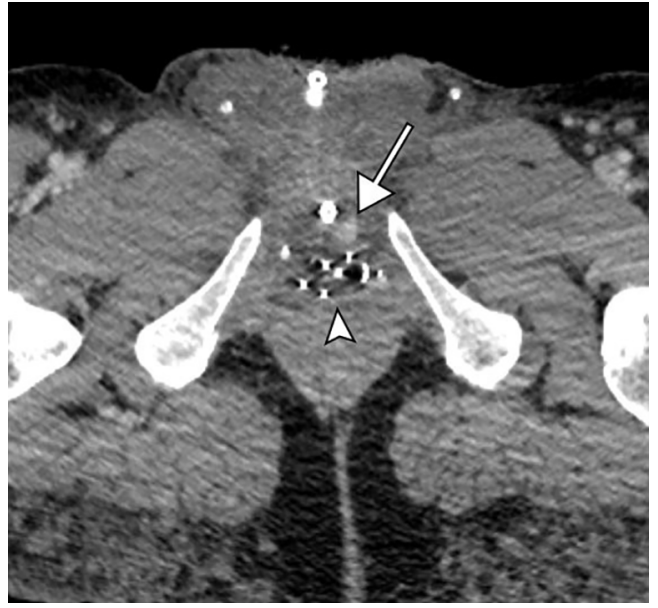

Figure 6 Urethral extravasation and neovaginal packing material. A 43-year-old transfeminine patient presented with hematuria days after penile inversion vaginoplasty. Contrast-enhanced computed tomography (CT) shows focus of active contrast extravasation at the left posterolateral aspect of the urethra (arrow), which demonstrated blooming on delayed images. Posteriorly, the neovagina is distended with packing material (arrowhead), which should not be misinterpreted as unintentionally retained foreign object.

Contrast-enhanced CT is the preferred modality for evaluation of abdominal or pelvic fluid collections (Figure 5). Abscesses appear as fluid filled collections with rim enhancement. Air may be present in some collections up to 2 weeks after surgery and is not diagnostic of superinfection. Superinfection of fluid collections is best judged by clinical and lab parameters, along with gram stain and culture of the aspirated fluid from these collections. It is helpful to instill rectal contrast material to help delineate normal adjacent bowel from potential fluid collections. CT can be used for image-guided drain placement via percutaneous approach in most patients. Early after surgery, the patient may still have neovaginal packing material or a dilator instrument that can simulate an abscess or a retained foreign object (Figure 6), so knowledge of this is helpful to avoid false positive diagnoses.

\section{Residual erectile tissue and prostate (I) Case example}

An asymptomatic 49-year-old transfeminine patient underwent pelvic MR after vaginoplasty (Figure 7). Incidentally noted was a large volume of residual erectile tissues. Although this patient had no symptoms or

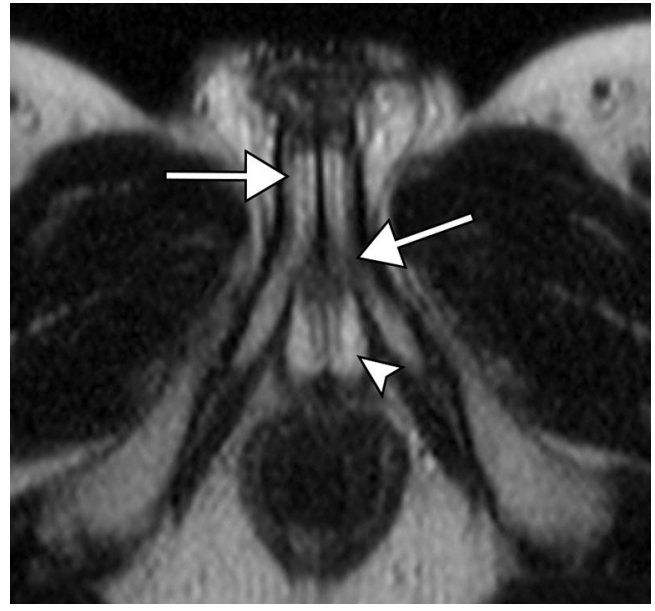

Figure 7 Residual erectile tissue. An asymptomatic 49-year-old transfeminine patient underwent pelvic magnetic resonance (MR) several years after penoscrotal inversion vaginoplasty. Axial T2weighted image incidentally showed T2-hyperintense residual corpora cavernosum (arrows) and spongiosum (arrowhead), consistent with residual erectile tissue.

complications related to the residual erectile tissue, it is important to note in case she might later develop bleeding, unexplained pelvic discomfort, or urethral stenosis.

\section{(II) Discussion}

Usually, residual erectile tissue is identified incidentally on CT or MRI with contrast as enhancing soft tissue at the base of the vagina. Vaginoplasty includes penile disassembly and resection of the corpora cavernosum and spongiosum. However, complete resection is not always possible and residual erectile tissues can lead to complications. In patients with persistent bleeding after surgery, residual erectile tissues are often implicated. The tissue may become engorged during sexual arousal and cause pain, fullness, or stenosis. The tissue may require re-excision if it is implicated as a cause for hemorrhage or pelvic discomfort. Also, the native prostate is left behind in patients undergoing transfeminine GS. This too may be mistaken for a mass on CT.

\section{Rectoneovaginal fistula \\ (I) Case example}

A 36-year-old transfeminine patient presented with neovaginal flatus and fecal discharge two weeks after PIV. She denied constitutional symptoms and pain. Physical exam showed fecal material in the neovaginal canal, but no obvious defect. What image modalities could be used to 


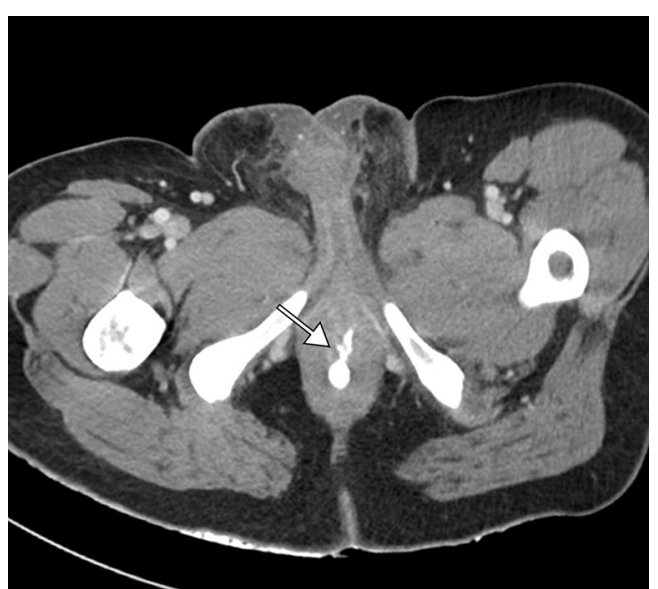

Figure 8 Rectoneovaginal fistula. A 36-year-old transfeminine patient presented with neovaginal flatus and fecal discharge 2 weeks after penoscrotal inversion vaginoplasty, suspicious for a rectoneovaginal fistula. Contrast-enhanced computed tomography (CT) with performed after instillation of iodinated rectal contrast material revealed a fistulous connection (arrow) between the anterior rectal wall and posterior wall of the neovagina. There was no deep pelvic fistula or abscess.

evaluate for suspected rectoneovaginal fistula?

\section{(II) Discussion}

Pelvic anatomy is best visualized at MRI given the soft tissue contrast availed by the modality; however, both CT and fluoroscopy can be useful for pre-surgical planning and to evaluate the extent of the lesion. Fluoroscopic studies such as a contrast enema may be considered first-line, but will not differentiate the surrounding structures well. CT and MR are ideally suited for evaluation of fistulas and allow for assessment of deep pelvic structures (Figure 8). Intracavitary contrast material (whether neovaginal or rectal) is recommended to help delineate the fistula tract when evaluating for fistulas. It is important to characterize the extent of the fistula and to exclude the potential for deep pelvic fluid collections or abscesses. Rectoneovaginal fistula is an uncommon complication of vaginoplasty and has been reported in $0.8-17.0 \%$ of cases $(2,14)$. Patient symptoms are quite suggestive and physical examination usually reveals the neovaginal defect. Patients at risk for rectoneovaginal fistulization can also be identified at imaging. The rectovaginal septum should have a minimum of thickness of about $5 \mathrm{~mm}$. If it measures thinner than this due to extensive pelvic dissection, this puts the patients at risk for fistula formation.

\section{Neovaginal or rectal prolapse \\ (I) Case example}

A 72-year-old transfeminine patient presented with progressive perineal fullness and pressure several years after undergoing revision vaginoplasty with colonic interposition. She denied symptoms of urinary or fecal retention. On physical examination, a tennis ball-sized bulge was noted in the perineum that was manually reducible, but recurred after manual pressure was removed. What imaging modality could be considered to adequately assess the extent of prolapse?

\section{(II) Discussion}

Dynamic MR imaging with defecography protocol allows for evaluation of the prolapsing structures and the degree and severity of prolapse. While most cases of prolapse are diagnosed by physical exam alone without imaging, cine pelvic MR with dynamic pelvic maneuvers (e.g., pelvic squeeze, Valsalva) can help to delineate the extent of prolapse and the relationship of the defect with the surrounding anatomy (Figure 9). On imaging, the organs prolapsing are seen extending below the pelvic outlet, either at rest or with provocative Valsalva maneuvers.

Pelvic dissection weakens the pelvic floor and these patients are at risk for prolapse. Neovaginal prolapse is an uncommon long-term complication after GS, occurring in up to $6 \%$ of patients after PIV and up to $8.0 \%$ of patients after intestinal vaginoplasty $(2,14)$. Prolapse is slightly more common after revision vaginoplasty. The complication may be prevented or treated by securing the neovaginal tube to the sacrospinous ligaments at the time of surgery.

\section{Conclusions}

Several imaging modalities are available to assist surgeons in the assessment of transgender patients for pre-operative assessment and with suspected complications after GS. Choosing the appropriate imaging modality and protocol modifications to align with clinical concerns can improve diagnostic accuracy. Working together, surgeons and radiologists can ensure accurate imaging assessment while 

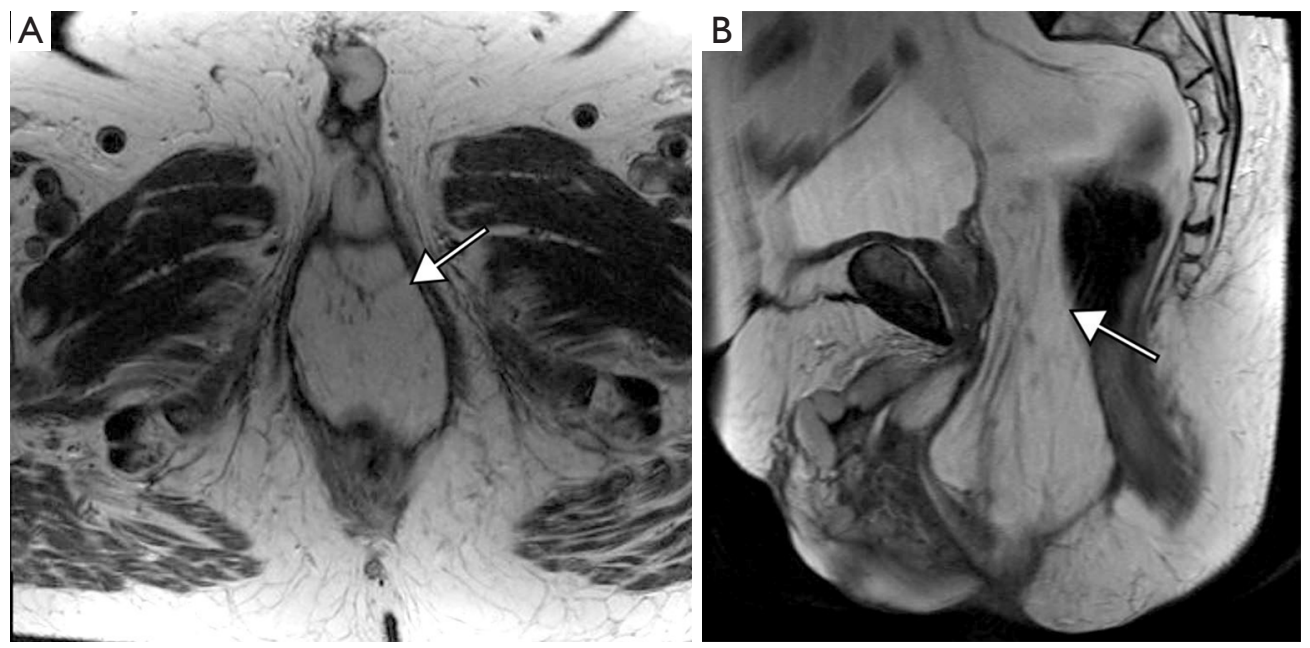

Figure 9 Prolapse (Video 1). A 72-year-old transfeminine patient complained of progressive perineal fullness and pressure several years after undergoing revision vaginoplasty with colonic interposition. (A) Axial magnetic resonance (MR) showed a T1- and T2-hyperintense mass (arrow) in the retroprostatic space. (B) Sagittal MR confirmed it to represent prolapsed mesenteric fat and vessels (arrow), extending well below the pelvic outlet.

accommodating for patient comfort.

\section{Acknowledgments}

Funding: None.

\section{Footnote}

Provenance and Peer Review: This article was commissioned by the Guest Editors (Drs. Oscar J. Manrique, John A Persing, and Xiaona Lu) for the series "Transgender Surgery" published in Annals of Translational Medicine. The article has undergone external peer review.

Conflicts of Interest: All authors have completed the ICMJE uniform disclosure form (available at http://dx.doi. org/10.21037/atm-20-6429). The series "Transgender Surgery" was commissioned by the editorial office without any funding or sponsorship. The authors have no other conflicts of interest to declare.

Ethical Statement: The authors are accountable for all aspects of the work in ensuring that questions related to the accuracy or integrity of any part of the work are appropriately investigated and resolved.

Open Access Statement: This is an Open Access article distributed in accordance with the Creative Commons Attribution-NonCommercial-NoDerivs 4.0 International License (CC BY-NC-ND 4.0), which permits the noncommercial replication and distribution of the article with the strict proviso that no changes or edits are made and the original work is properly cited (including links to both the formal publication through the relevant DOI and the license). See: https://creativecommons.org/licenses/by-nc-nd/4.0/.

\section{References}

1. Stowell JT, Horowitz JM, Thomas S. Gender-affirming surgical techniques, complications, and imaging considerations for the abdominal radiologist. Abdom Radiol (NY) 2020;45:2036-48.

2. Stowell JT, Grimstad FW, Kirkpatrick DL, et al. Imaging Findings in Transgender Patients after Gender-affirming Surgery. Radiographics 2019;39:1368-92.

3. Nikolavsky D, Hughes M, Zhao LC. Urologic Complications After Phalloplasty or Metoidioplasty. Clin Plast Surg 2018;45:425-35.

4. Ascha M, Massie JP, Morrison SD, et al. Outcomes of Single Stage Phalloplasty by Pedicled Anterolateral Thigh Flap versus Radial Forearm Free Flap in Gender Confirming Surgery. J Urol 2018;199:206-14.

5. Sinove Y, Kyriopoulos E, Ceulemans P, et al. Preoperative planning of a pedicled anterolateral thigh (ALT) flap for 
penile reconstruction with the multidetector CT scan. Handchir Mikrochir Plast Chir 2013;45:217-22.

6. Morrison SD, Perez MG, Carter CK, et al. Pre- and Post-Operative Care With Associated Intra-Operative Techniques for Phalloplasty In Female-to-Male Patients. Urol Nurs 2015;35:134-8.

7. Blaschke E, Bales GT, Thomas S. Postoperative imaging of phalloplasties and their complications. AJR Am J Roentgenol 2014;203:323-8.

8. Schardein JN, Zhao LC, Nikolavsky D. Management of Vaginoplasty and Phalloplasty Complications. Urol Clin North Am 2019;46:605-18.

9. Dabela-Biketi A, Mawad K, Li H, et al. Urethrographic Evaluation of Anatomic Findings and Complications after Perineal Masculinization and Phalloplasty in Transgender Patients. Radiographics 2020;40:393-402.

10. Monstrey S, Hoebeke P, Selvaggi G, et al. Penile

Cite this article as: Stowell JT, Metcalfe AM, Jha P. Imaging evaluation for the diagnosis and management of complications of gender-affirming surgeries. Ann Transl Med 2021;9(7):609. doi: $10.21037 /$ atm-20-6429 reconstruction: is the radial forearm flap really the standard technique?. Plast Reconstr Surg 2009;124:510-8.

11. Kang A, Aizen JM, Cohen AJ, et al. Techniques and considerations of prosthetic surgery after phalloplasty in the transgender male. Transl Androl Urol 2019;8:273-82.

12. Hoebeke PB, Decaestecker K, Beysens M, et al. Erectile implants in female-to-male transsexuals: our experience in 129 patients. Eur Urol 2010;57:334-40.

13. Selvaggi G, Hoebeke P, Ceulemans P, et al. Scrotal reconstruction in female-to-male transsexuals: a novel scrotoplasty. Plast Reconstr Surg 2009;123:1710-8.

14. Manrique OJ, Adabi K, Martinez-Jorge J, et al. Complications and Patient-Reported Outcomes in Male-to-Female Vaginoplasty-Where We Are Today: A Systematic Review and Meta-Analysis. Ann Plast Surg 2018;80:684-91. 